Revista Brasileira de Farmacognosia Brazilian Journal of Pharmacognosy 21(5): 908-914, Sep./Oct. 2011

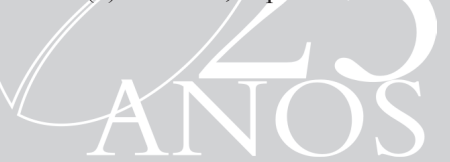

Article

Received 14 Jan 2011

Accepted 23 Mar 2011

Available online 2 Sep 2011

Keywords:

essential oil

Leishmania amazonensis

nerolidol

Piper claussenianum

ISSN 0102-695X

http://dx.doi.org/10.1590/S0102-

$695 \times 2011005000157$

\section{Antileishmanial activity of nerolidol-rich essential oil from Piper claussenianum}

\author{
André Mesquita Marques, ${ }^{1}$ Anna Léa Silva Barreto, ${ }^{2}$ José \\ Alexandre da R. Curvelo, ${ }^{2}$ Maria Teresa Villela Romanos, ${ }^{2}$ \\ Rosangela M. de A. Soares, ,'2 Maria Auxiliadora C. Kaplan ${ }^{1}$
}

\author{
${ }^{l}$ Núcleo de Pesquisas de Produtos Naturais, Centro de Ciências da Saúde, \\ Universidade Federal do Rio de Janeiro, Brazil, \\ ${ }^{2}$ Departamento de Microbiologia Geral, Instituto de Microbiologia Paulo Góes, \\ Universidade Federal do Rio de Janeiro, Brazil.
}

\begin{abstract}
Leishmaniasis is one of the most neglected tropical diseases, representing a group of parasitic diseases worldwide spread, occurring in 88 tropical and subtropical countries. Approximately 350 million people live in areas of active transmission of leishmaniasis, with about 1-2 million estimated new cases occurring every year. More than $90 \%$ of the cutaneous cases appear in developing countries. Efforts to improve the therapeutic arsenal against leishmaniasis have led to the search for new and cheap range of drugs. In this study, the nerolidol-rich essential oil from Piper claussenianum (Miq.) C. DC., Piperaceae, was assayed on arginase activity of Leishmania amazonensis. The effect of this essential oil on arginase activity levels showed an enzyme inhibition of $62.2 \%$. This result stimulates the scientific interest about the potential value of this plant species on neglected diseases as potential new natural product source of pharmacological interest for the treatment of leishmaniasis.
\end{abstract}

\section{Introduction}

Leishmaniasis is one of the most neglected tropical diseases, due to few tools available for controlling and also the lack of clear criteria for the disease management. Leishmaniasis represents a group of parasitic diseases worldwide spread, occurring in 88 tropical and subtropical countries. Approximately 350 million people live in areas of active transmission of Leishmania, as many as 12 million people are believed to be currently infected, with about 1-2 million estimated new cases occurring every year throughout Africa, Asia, Europe, and the Americas directly affected by leishmaniasis. More than $90 \%$ of the cutaneous cases appear in developing countries (Desjeux et al., 2004; WHO 2007). This illness can be promoted by more than twenty species of trypanosomatids from the genus Leishmania. The parasite is transmitted by insect vectors of the genera Lutzomyia and Phlebotomus (Diptera: Psychodidae: Phlebotominae), and is able to affect animals and humans (Ashford et al., 2000). The cutaneous leishmaniasis (CL) has afflicted humankind for centuries, and represent an infection characterized by a heteroxenic cycle affecting mainly skin or mucous membranes and is distinguished by the presence of ulcerative lesions. CL may cause the impairment and destruction of mucosal and cutaneous structures, frequently producing facial mutilation (Murray et al., 2004). Since the discovery of the first class of drugs able to treat leishmaniasis (i.e., pentavalent antimonials), the search for substances with antileishmanial activity, without toxic effects, and able to overcome the emergence of drug resistant strains still remains a current goal (Santos et al., 2008). Efforts to improve therapeutic arsenal against leishmaniasis have led to the search for new and cheap range of drugs. The searching for new drugs with antileishmanial activities, including natural product compounds, have been undertaken worldwide, although problems with the side effects of the chemotherapies currently used have not yet been solved (Santin et al., 2009). Natural products have been widely employed in folk medicine to treat infectious diseases, mainly in communities with inadequate conditions of health and sanitation. Many studies have shown plants of many regions of the world containing compounds with anti-protozoal activity. It has been reported that various essential oils (EO) show inhibitory action against diverse human parasites related to neglected tropical diseases such as L. amazonensis (Marques et al., 2010; Silva et al., 2009; Ueda-Nakamura et al., 2006).

In this work a nerolidol-rich essential oil from leaves of Piper claussenianum (Miq.) C. DC., Piperaceae, 
was tested against L. amazonensis enzymatic activity of arginase, in order to understand the growth inhibition mechanism of this EO over this specific parasite, which has been previously reported elsewhere (Marques et al., 2010).

\section{Materials and Methods}

Plant material and essential oil extraction

Leaves $(100 \mathrm{~g})$ of Piper claussenianum (Miq.) C. DC., Piperaceae, were collected in Castelo-ES in January 2010. The botanical vouchers were identified by Dr. Elsie Franklin Guimarães and kept at the Herbarium (HB) of the Rio de Janeiro Botanical Garden (JBRJ), registered under number RB 489043. The fresh and dried plant materials were submitted to hydrodistillation for 2 $\mathrm{h}$ in a modified Clevenger-type apparatus. The obtained essential oils (EO) were dried over anhydrous sodium sulphate, yielding $1 \% \mathrm{w} / \mathrm{v}$ in both studied specimens.

\section{GC-FID analysis}

The gas chromatography analyses were carried out on a GC 2010 Shimadzu with a ZB-1MS fused silica capillary column $(30 \mathrm{~m} \times 0.25 \mathrm{~mm} \times 0.25 \mu \mathrm{m}$ film thickness). The operating temperatures used were as follows: injector $260{ }^{\circ} \mathrm{C}$, detector $290{ }^{\circ} \mathrm{C}$ and column oven $60{ }^{\circ} \mathrm{C}$ up to $290{ }^{\circ} \mathrm{C}\left(10^{\circ} \mathrm{C} / \mathrm{min}\right)$. Hydrogen at 1.0 $\mathrm{mL}$ min-1 was used as a carrier gas. The percentages of the compounds were obtained by GC-FID analysis.

\section{GC-MS analysis}

Qualitative analyses were carried out on a GCQP2010 PLUS Shimadzu with a ZB-5MS fused silica capillary column $(30 \mathrm{~m} \times 0.25 \mathrm{~mm} \times 0.25 \mu \mathrm{m}$ film thickness) under the experimental conditions reported for GC-FID analysis. The essential oil components were identified by comparing their retention indices and mass spectra to published data and computer matching with WILEY 275 and the National Institute of Standards and Technology (NIST 3.0) libraries provided by a computer-controlled GC-MS system. The results were also confirmed by comparing the compounds' elution order with their relative retention indices reported in the literature (Adams, 2001). The retention indices were calculated for all the volatile constituents using the retention data of linear $n$-alkanes $\mathrm{C}_{8}-\mathrm{C}_{24}$.

\section{Nuclear magnetic resonance spectroscopy}

The EO obtained was analysed by ${ }^{1} \mathrm{H}$ and ${ }^{13} \mathrm{C}$ NMR and recorded on a Brüker DRX 400 spectrometer. The chemical shifts were determined in $\mathrm{CDCl}_{3}$, using
TMS as the internal standard. The spectrometric data for the ${ }^{1} \mathrm{H}$ NMR spectra are organised in agreement with the convention: chemical shift (number of protons and multiplicity), and the data for ${ }^{13} \mathrm{C}$ NMR spectra are organised by chemical shift. The signals of the NMR analyses were compared to the literature. (Marques et al., 2010).

\section{Biological assays}

Parasite

One L. amazonensis strain (MHOM/BR/75/ Josefa) originally isolated from a human CL, kindly provided by Dr Elvira Saraiva (UFRJ, Brazil) was used in this study. Infective promastigote forms were maintained by weekly transfers in $25-\mathrm{cm}^{2}$ culture flasks with Schneider's insect medium (SIM) (Sigma Aldrich ${ }^{\circledR}$ St. Louis/ USA), pH 7.2, supplemented with $10 \%$ fetal bovine serum (FBS) and Gentamicine $(80 \mu \mathrm{g} / \mathrm{mL})$ at 26 ${ }^{\circ} \mathrm{C}$. By this method, a five passage strain was used in all experiments carried out.

\section{Protozoal arginase activity}

Arginase enzyme activity was measured as previously described by Kropf et al. (2005), with slight modifications. Promastigotes were incubated for $72 \mathrm{~h}$ at $26{ }^{\circ} \mathrm{C}$ in presence or absence of the P. claussenianum leaves essential oil IC50 (Marques et al., 2010). Inhibition concentrations previously tested (Marques et al., 2010) was used in all experiments, since the EO stock solution was obtained by a single isolation and extraction. After incubation, $10^{8}$ cells were mixed with $100 \mu \mathrm{L}$ of $0.1 \%$ Triton $\mathrm{X}-100$ and underwent five cycles of $3 \mathrm{~min}$ in the vortex and $1 \mathrm{~min}$ on ice bath. Thereafter, $100 \mu \mathrm{L}$ of $25 \mathrm{mM}$ Tris- $\mathrm{HCl} \mathrm{pH} 8.3$ were added to the sample. For arginase activity measurements, $10 \mu \mathrm{L}$ of 10 $\mathrm{mM} \mathrm{MnCl}{ }_{2}$ were added to each $100 \mu \mathrm{L}$ of the lysate. The arginase was then activated by heating the sample for 10 min at $56^{\circ} \mathrm{C}$, and arginine hydrolysis was conducted by incubated the sample with $100 \mu \mathrm{L}$ of $0.5 \mathrm{M}$ L-arginine, $\mathrm{pH} 9.7$ at $37{ }^{\circ} \mathrm{C}$ for $20 \mathrm{~min}$. The reaction was stopped by adding $800 \mu \mathrm{L}$ of $\mathrm{H}_{2} \mathrm{SO}_{4}(96 \%), \mathrm{H}_{3} \mathrm{PO}_{4}(85 \%)$ and $\mathrm{H}_{2} \mathrm{O}$ $(1 / 3 / 7, v / v / v)$ to the sample and mixing with $40 \mu \mathrm{L}$ of $4 \%$ of $\alpha$-isonitrosopropiophenone (dissolved in ethanol). After heating $\left(95^{\circ} \mathrm{C}\right.$ for $\left.30 \mathrm{~min}\right)$, urea concentration was determined by spectrometry at $540 \mathrm{~nm}$ wavelenght.

\section{Interaction Leishmania-mammal host cells}

To assess the influence of IC50 P. claussenianum leaves essential oil on parasite host cell interactions, parasites were treated or not for $1 \mathrm{~h}$ with the EO, centrifuged at $4500 \mathrm{rpm}$, at $4{ }^{\circ} \mathrm{C}$ for $3 \mathrm{~min}$, washed twice 
in $10 \mathrm{mM}$ PBS pH 7.2 and ressuspended in cold sterile Dulbecco's modified Eagle's medium (DMEM) $\left(\mathrm{GIBCO}^{\circledR}\right)$ to adjust the inoculum to $5 \times 10^{6}$ cells. Coverslips adhered macrophages, obtained from BALB/c mice peritoneal washing, were incubated with the parasites, in a ratio of 10 parasites per host cell, in $300 \mu \mathrm{L}$ of DMEM supplemented with $2 \%$ fetal calf serum at $37{ }^{\circ} \mathrm{C} 5 \% \mathrm{CO}_{2}$ for an hour. After interaction, the material was fixed in methanol for $5 \mathrm{~min}$, washed and stained with Giemsa $36 \%$ for $40 \mathrm{~min}$. Thereafter, coverslips were dehydrated in acetone and xylene, in concentrations 90:10, 70:30, $30: 70$ and 0:100 respectively, and stuck to a blade $26 \mathrm{x}$ 76 with resin Entellan ${ }^{\circledR}$.

\section{Nitrite determination}

Nitrite is a stable oxidation product of nitric oxide (NO), that can be measured spectrophotometricaly by adding Griess reagent $(0.1 \%$ sulfanilamide and $0.1 \%$ $\mathrm{N}-1$ naphtylethylenediamine in $2.5 \%$ phosphoric acid) at the same volume of infected macrophages cultures supernatants. After $20 \mathrm{~min}$ at room temperature and in the dark, the absorbance of the chromophore was measured at $540 \mathrm{~nm}$ wavelenght. Nitrite concentrations were estimated by comparison with a standard curve prepared with sodium nitrite in DMEM supplemented with $10 \%$ fetal bovine serum (v/v). Positive control of NO production, were macrophages treated with INF $\gamma(50$ $\mathrm{ng} / \mathrm{mL}$ ) or treated with INF $\gamma$ and the EO.

\section{Cytotoxicity assay}

To evaluate the possible essential oil's toxicity to mammal cells, a cytotoxicity assay was performed using L929 fibroblast cells (mouse) and Raw cells (mouse macrophages). The cells were grown in Eagle's minimum essential medium (Eagle-MEM) supplemented with $10 \%(\mathrm{v} / \mathrm{v})$ fetal bovine serum, glutamine $(0.03 \mathrm{mg} /$ $\mathrm{mL})$, garamycin $(50 \mu \mathrm{g} / \mathrm{mL})$, fungizone (amphotericin B) $(2.5 \mathrm{mg} / \mathrm{mL}), \mathrm{NaHCO}_{3}(0.25 \%)$ and HEPES $(10 \mathrm{mM})$ (Schmidt, 1979). Cell cultures were prepared in 96-well microtiter plates (Falcon Plastics, Oxnard, CA, USA) and incubated at $37{ }^{\circ} \mathrm{C}$ in a $5 \% \mathrm{CO}_{2}$ atmosphere.

Cytotoxicity was evaluated by the neutral red dye-uptake method (Neyndorff et al., 1990). To determine the cytotoxicity of the $P$. claussenianum essential oil extracted from leaves, several concentrations of each sample (range from 40 to $0.56 \mathrm{mg} / \mathrm{mL}$ ) were placed in contact with confluent L929 fibroblast cell monolayers and incubated at $37{ }^{\circ} \mathrm{C}$ in a $5 \% \mathrm{CO}_{2}$ atmosphere for 24 h. After incubation, cells were incubated in the presence of $0.01 \%$ neutral red solution for $3 \mathrm{~h}$ at $37{ }^{\circ} \mathrm{C}$ in a $5 \%$ $\mathrm{CO}_{2}$ atmosphere. Then, the medium was removed and the cells were fixed with $4 \%$ formalin in phosphate-buffered saline, $\mathrm{pH}$ 7.2. The dye incorporated by the viable cells was eluted using a mixture of methanol: acetic acid: water (50:1:49), and the dye uptake was determined by measuring the optical density (OD) of the eluate at 490 $\mathrm{nm}$ in an automatic spectrophotometer (ELx800TM-BioTeK Instruments, Inc.). The $50 \%$ cytotoxic concentration (CC50) was defined as the concentration that caused a $50 \%$ reduction in dye uptake.

All experiments were performed in triplicate, and the statistical analysis of the differences between mean values obtained was done by Student's t test. $p$ values of 0.05 or less were considered significant.

\section{Results and Discussion}

The essential oils obtained by hydrodistillation from leaves of $P$. claussenianum in fresh and in dried material yielded about $1 \%(\mathrm{w} / \mathrm{v})$ to both cases. Results for the qualitative and quantitative analysis of the essential oils are shown in Table 1 for the leaf volatile constituents. In total, sixteen different compounds were identified, ranging from $92.48 \%$ and $97.72 \%$ of the oils for fresh and dried leaves respectively. The oils showed a rich sesquiterpene content. Sesquiterpenes were identified as the main volatile fraction of the leaves, being Nerolidol the major constituent, ranging of up to $81.0 \%$ in EO obtained by hydrodistillation from fresh and dried samples. The structure of nerolidol was confirmed by GC-MS, ${ }^{1} \mathrm{H}$ NMR and ${ }^{13} \mathrm{C}$ NMR spectra (Marques et al., 2010). The oil from leaves of $P$. claussenianum was characterized by their high content in (E)-nerolidol (81.41; $83.29 \%)$ as well as linalool $(5.20 ; 2.15 \%), \gamma$-muurolene (1.09; 3.22\%), (E)-caryophyllene $(0.64 ; 1.40 \%)$ and $\gamma$-elemene $(0.54 ; 0.78 \%)$ fresh and dried material, respectively. The high percentage of nerolidol in EO from leaves of $P$. claussenianum allowed us to perform biological assays against $L$. amazonensis to understand the mechanism of growth inhibition of this parasite in cutaneous leishmaniasis. The fresh leaf EO with $81.41 \%$ of nerolidol was used to perform the biological assays on arginase inhibition.

Arginase is a dependent manganese metalloenzyme able to hydrolyze L-arginine to L-ornithine and urea, and its activity has been related to the synthesis of nitric oxide (NO), and therefore associated to cytotoxic process and immunological mechanisms (Kanyo et al., 1996; Da Silva et al, 2002). The arginase activity in the Tripanossomatidae family has been shown in some specific genre, and it has been used for identification and classification purposes. The genus Leishmania has a detectable arginase activity which is considered to have a role in the production of ornithine reported by Da Silva et al. (2002) and therefore essential for the growth of the parasite, since previous studies revealed that arginase null protozoans are unable to generate polyamines (Vincendeau et al., 
2003; Roberts et al., 2004).

The effect of the leaf essential oil of $P$. claussenianum showed $62.17 \%$ inhibition in the levels of arginase activity (Figure 1). Regarding to the Leishmania-macrophage interaction mechanisms, when parasites were pretreated for one hour with the IC50 of the EO from leaves of $P$. claussenium, the infected macrophages percentage was reduced in $42.7 \%(p=0,01)$. When the EO was used on infected macrophages the infected macrophages percentage was reduced in $31.25 \%$ $(p=0,03)$ (Figure 2). The production of NO from infected macrophages treated for $24 \mathrm{~h}$ with the IC50 of the EO was measured by the nitrite concentration in the culture supernatants, using Griess reagent. In this case there was an increase of $17.2 \%$ in NO production, an increase greater than that observed when infected cells were treated with INF- $\gamma$. Interestingly an increase of $20,5 \%$ in NO production could be seen when EO and INF- $\gamma$ were used together (Figure 3).

Table 1. Identified compounds in the essential oil from leaves of $P$. claussenianum.

\begin{tabular}{lccccc}
\hline \multicolumn{1}{c}{ Compounds } & ${ }^{2}{ }^{2}{ }^{\mathrm{Lit}}$ & ${ }^{\mathrm{b} R I}$ & $\begin{array}{c}\text { Fresh } \\
\text { H.D } \%\end{array}$ & $\begin{array}{c}\text { Dry } \\
\text { H.D } \%\end{array}$ & Identification \\
\hline$\alpha$-pinene & 939 & 936 & 0.17 & - & RI, GCMS \\
$\beta$-pinene & 979 & 976 & 0.18 & 0.15 & RI, GCMS \\
$\beta$-myrcene & 991 & 987 & - & 0.18 & RI, GCMS \\
$(Z$ )- $\beta$-ocimene & 1037 & 1033 & 0.62 & - & RI, GCMS \\
$(E$ )- $\beta$-ocimene & 1050 & 1043 & 0.85 & - & RI, GCMS \\
cis-linalool oxide & 1087 & 1083 & 0.57 & - & RI, GCMS \\
linalool & 1097 & 1101 & 5.20 & 2.15 & RI, GCMS \\
$\gamma$-elemene & 1338 & 1340 & 0.12 & 1.79 & RI, GCMS \\
$\beta$-elemene & 1391 & 1387 & 0.54 & 0.78 & RI, GCMS \\
$(E)$-caryophyllene & 1419 & 1415 & 0.64 & 1.40 & RI, GCMS \\
$\alpha$-humulene & 1455 & 1451 & 0.59 & 1.09 & RI, GCMS \\
$\gamma$-muurolene & 1480 & 1477 & 1.09 & 3.22 & RI, GCMS \\
$\alpha$-selinene & 1498 & 1497 & 0.35 & - & RI, GCMS \\
$\delta$-cadinene & 1523 & 1520 & 0.38 & 0.76 & RI, GCMS \\
$(E)$-nerolidol* & 1563 & 1563 & 81.41 & 83.29 & RI, GCMS \\
$\alpha$-eudesmol & 1654 & 1654 & 0.50 & 2.91 & RI, GCMS \\
\% Peak sum of identified compounds & 92.48 & \multicolumn{2}{c}{97.72} \\
\hline
\end{tabular}

${ }^{a}$ RI Lit: Literature Retention Indices; ${ }^{\mathrm{b}} \mathrm{RI}$ : Experimental Retention Indices; H.D: Hydrodistillation; *The structure of $(E)$-nerolidol was also confirmed by NMR and Internal Standard FID analysis.

The effect of essential oil of $P$. claussenianum on arginase activity levels showed a strong and significant inhibition of the enzyme activity. The ability to survive and multiply within macrophages is a particular feature of several microorganisms including Trypanossoma cruzi and Leishmania. In order to sustain a chronic infection, parasites must subvert macrophage-accessory cell activities and ablate the development of protective immunity
(Alexander et al., 2002). The increase in NO production by macrophages infected and treated with the EO suggests that it may be considered a useful strategy in controlling the infection by inhibiting parasite arginase.

The most important mechanism for the killing of Leishmania and for the control of leishmaniasis is the production of nitric oxide by macrophages (Holzmuller et al., 2006). These immune cells can control Leishmania infection when Th1 response is mounted and proinflammatory cytokines are released. This process leads to the induction of inducible nitric oxide sinthase (iNOS) and NO production. Arginine, a substrate of NO production, is modulated by both Th1 and Th2 responses in a manner that Th1 response increases conversion of arginine to NO, whereas the Th2 response promotes arginase induction (Wanasen \& Soong, 2008).

The inhibition of the enzymatic activity may be able to modulate cellular infection in Leishmania major and Leishmania infantum infected macrophages, while the increasing parasite arginase activity by IL-4 induction promotes Leishmania growth (Iniesta \& GomesNieto, 2001). Futhermore, Iniesta and colleagues in 2005 demonstrated that L. major infection leads to arginase I induction in macrophages, and that host arginase I induction supports Leishmania growth.

Previous studies have demonstrated that the arginase activity in Leishmania spp. is able to modulate the nitric oxide synthase activity (Boucher et al., 1999; Wanasen \& Soong, 2008). Besides, studies using models of visceral leishmaniasis by $L$. donovani showed that the parasite is able to modulate the activity of arginase in spleen cells of infected animals, increasing the enzyme expression and favoring the maintenance of infection (Osorio et al., 2008). Strikingly, the active role played by Leishmania arginase in diverting arginine away from the iNOS pathway was demonstrated by the increased host NO response to L. mexicana knockout for arginase infection in mice. The L. mexicana knockout for arginase infection led to an enhanced Th1 response associated to an INF- $\gamma$ upregulation as well. Taken together, these responses led to a significant growth attenuation of the parasite in mice (Gaur et al., 2007). Considering the importance of the cellular signaling pathways involving the host cells and parasites of the Leishmania genus there are few studies in the literature connecting the importance of the parasite arginase inhibition and macrophage NO production. Therefore, arginase-specific inhibitors may be considered a potential alternative among new substances with pharmacological interest for the treatment of leishmaniasis.

The interference in Leishmania - mammalian host cell interaction suggests that the essential oil of $P$. claussenianum may action on early steps of this process of interaction. Therefore this EO may be able to modulate the virulence factors not yet identified, or to block specific 


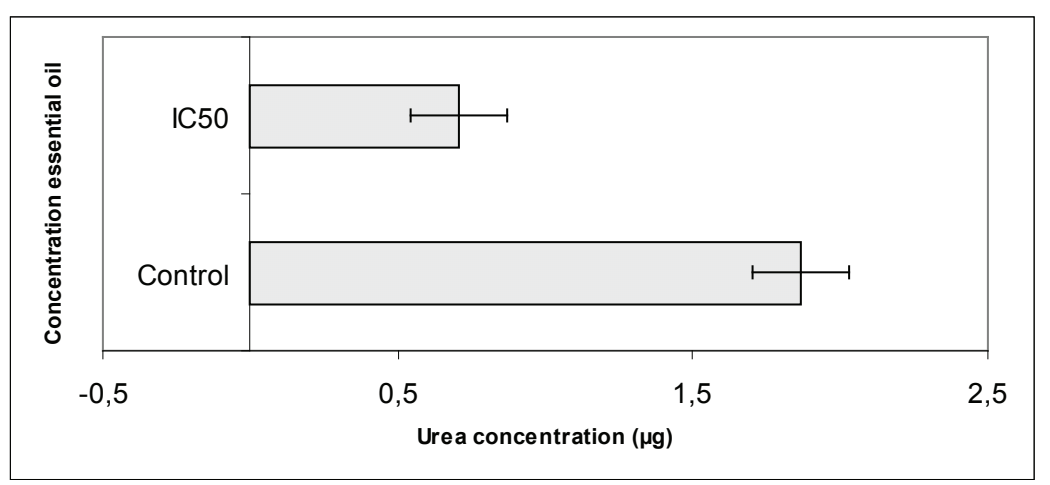

Figure 1. Essential oil from leaves of P. claussenianum in arginase activity of promastigotes of L. amazonensis. The cells were incubated in the absence or presence of IC50 for $72 \mathrm{~h}$ at $26^{\circ} \mathrm{C}$. The enzyme activity was measured by the concentration of urea in the system $(p=0.03)$.
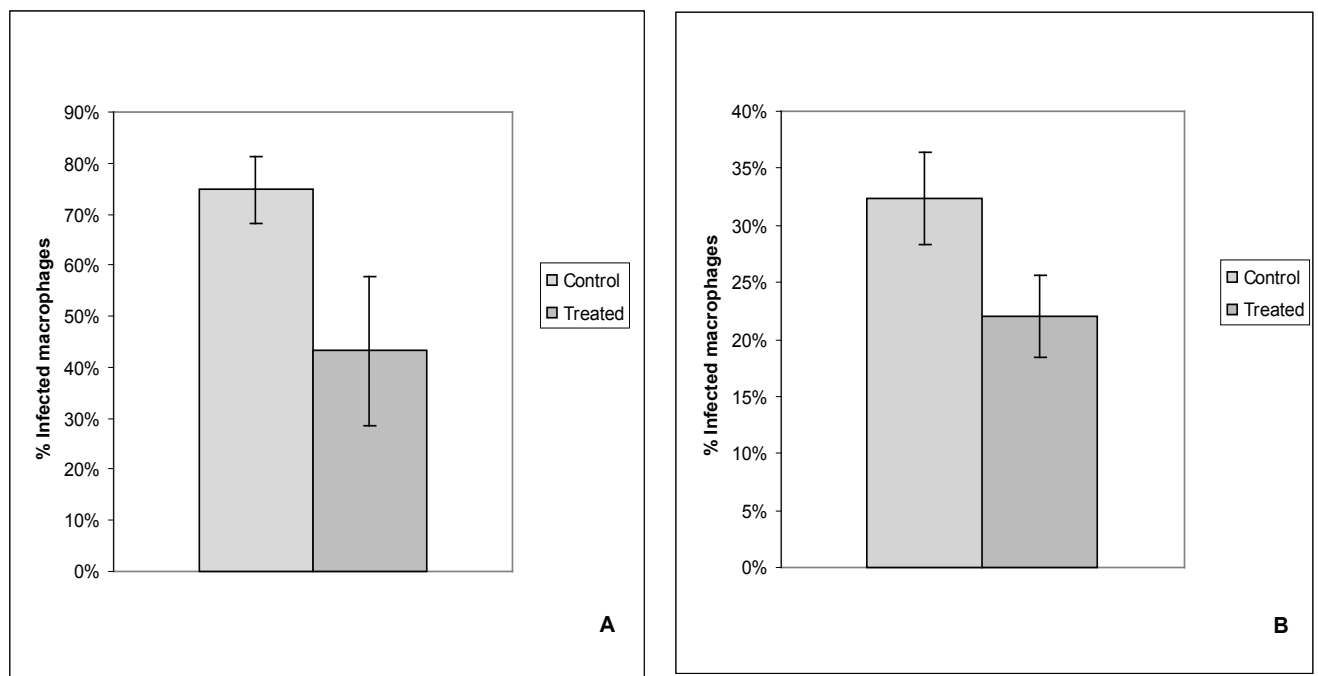

Figure 2. A. Percentage of macrophages infected with L. amazonensis pretreated or not with the IC50 of essential oil from leaves of $P$. claussenianum after one hour of interaction. Reduction of $42.7 \%$ infection $(p=0.01)$. B. Percentage of macrophages infected with $L$. amazonensis treated or not with the IC50 of essential oil from leaves of $P$. claussenianum for 24 h. Reduction of $31.25 \%$ of infection $(p=0.03)$.

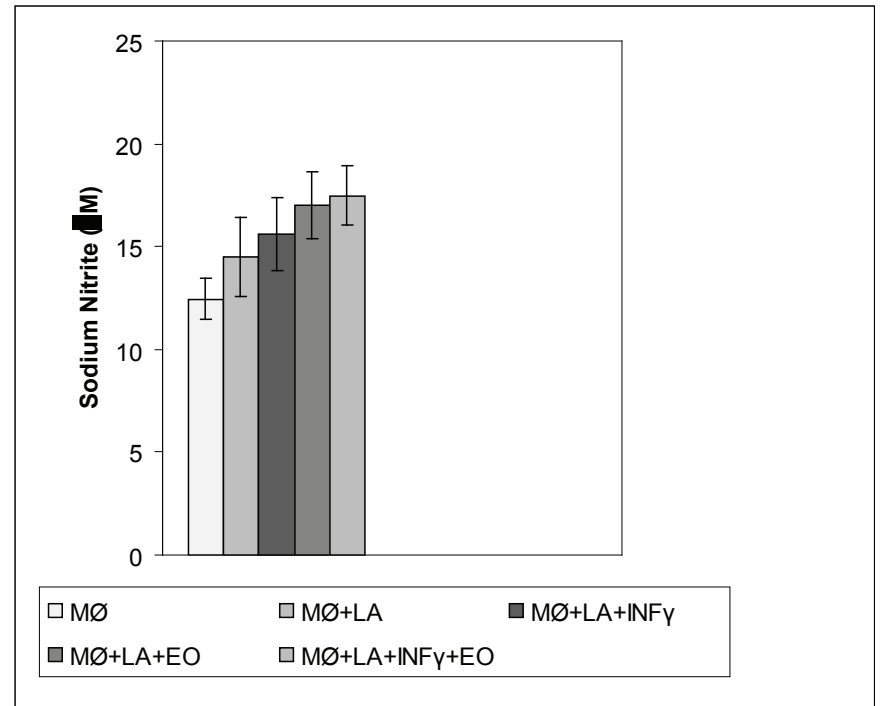

Figure 3. Nitrite dosages of culture supernatants of macrophages (MØ) infected with L. amazonensis and treated with the IC50 of the EO for $24 \mathrm{~h}$. As controls were used uninfected and infected MØ treated with INF- $\gamma$. 
receptors. This experiment was carried out with subinhibitory concentration of the EO in order to avoid compromising the viability of parasites. Santos et al. (2009) observed similar results when L. amazonensis promastigotes were pre-treated with protease inhibitors for HIV.

The citotoxicity assay revealed that the EO extracted from leaves of $P$. claussenianum has no toxicity effect to fibroblasts nor macrophages cell lines in any concentration tested (ranging from 40 to $0.56 \mathrm{mg} / \mathrm{mL}$ ). Macrophages and fibroblasts generate from mice were challenged with the essential oil extracted from the leaves of $P$. claussenianum (range 40 to $0.56 \mathrm{mg} / \mathrm{mL}$ ), whose major component is $(E)$-nerolidol, demonstrating no cytotoxic effect on either cell lines in any concentration. This finding agrees with literature data, in fact toxic effects were not observed in vivo after nerolidol intraperitonial or topic administration in mouse (Arruda at al., 2005). In vivo studies have demonstrated that animals treated with nerolidol in concentrations up to $200 \mathrm{mg} / \mathrm{kg}$ showed no clinical signs or morbidity, and in addition, peripheral blood cells and liver taken from these animals showed no DNA damage (Pículo et al., 2010). Thus, the absence of citotoxicity in high concentrations may be considered a relevant issue pointing to the possible administration of this compound as an alternative for the management of leishmaniasis, since it is able to control protozoa growth, as well as modulate some crucial virulence factors, such as the arginase activity and the interaction mechanisms between parasites and mammal host cells.

\section{Conclusion}

The essential oil extracted from the leaves of $P$. claussenianum is a natural product rich in $(E)$-nerolidol that has a surprising biological activity, showing efficient growth inhibition of promastigote forms of $L$. amazonensis, and a significant inhibition on its arginase activity as well as an important enhancement in the NO levels in infected macrophages induced by this EO. Moreover, this essential oil is able to interfere with the parasite-host cell interaction, reducing the percentage of infected cells. Nevertheless, no cytotoxic effect was evidenced against any of the mammalian cells lines tested. The results presented in this study points to the $P$. claussenianum leaves essential oil as an important source in the searching for new alternatives for the treatment and management of cutaneous leishmaniasis.

\section{Acknowledgments}

The authors would like to thank to the National Council for Scientific and Technological Development for financial support.

\section{References}

Adams RP 2001. Identification of essential oil components by gas chromatography/quadrupole mass spectrometry. Ilinois: Allured Publishing Corporation.

Alexander J, Satoskar AR, Russel DG 2002. Leishmania species: models of intracellular parasitism. J Cell Sci 112: 2993-3002.

Arruda DC, Alexandri FL, Katzin AM, Uliana SRB 2005. Antileishmanial activity of the terpene nerolidol. Antimicrob Agents Ch 49: 1679-1687.

Ashford RW 2000. The leishmaniasis as emerging and reemerging zoonoses. Int J Parasitol 30: 1262-1281.

Boucher JL, Moali C, Tenu JP 1999. Nitric oxide biosynthesis, nitric oxide synthase inhibitors and arginase competition for L-arginine utilization. Cell Mol Life Sci 55: 10151028.

Da Silva ER, Castilho TM, Pioker FC, Tomich de Paula CH, Floeter-Winter LM 2002. Genomic organization and transcription characterization of the gene encoding Leishmania (Leishmania) amazonensis arginase and its protein structure prediction. Int J Parasitol 32: 727 737.

Desjeux P 2004. Leishmaniasis: current situation and new perspectives. Comp Immunol Microbiol Infec Dis 27: 305-318.

Gaur U, Robert SC, Dalvi RP, Corraliza I, Ullman B 2007. An effect of parasite-encoded arginase on the outcome of murine cutaneous Leishmaniasis. J Immunol 179: 84468453.

Holzmuller P, Gonçalves R, Lemersre JL 2006. Phenotipycal characteristics, biochemical pathways, molecular targets and putative role of nitric oxide-mediated programmed cell death in Leishmania. Parasitology 132: 19-32.

Iniesta V, Gomes-Nieto LC, Corrraliza I 2001. II The inhibition of arginase by $\mathrm{N}$ (omega)-hydroxy-1-arginine controls the growth of Leishmania inside macrophages. $J$ Exp Med 193: 777-784.

Iniesta V, Carcelen J, Molano I, Peixoto PM, Redondo E 2005. Arginase I induction during Leishmania major infection mediate de development of disease. Infect Immun 73: 6085-6090.

Kanyo ZF, Scolnick LR, Ash DE, Christianson DW 1996. Structure of a unique binuclear manganese cluster in arginase. Nature 383: 554-557.

Kropf P, Fuentes JM, Fahnrich E, Arpa L, Herath S, Weber V, Soler G, Selada A, Modolell M, Muller I 2005. Arginase and polyamine synthesis are key factors in the regulation of experimental leishmaniasis in vivo. FASEB J 19: 1000-1002.

Marques AM, Barreto ALS, Curvelo JAR, Velozo LSM, Moreira DL, Guimarães EF, Soares RMA, Kaplan MAC 2010. Biological activity and chemistry of essential oils from Piper claussenianum essential oil (Piperaceae). Nat Prod Commun 11: 1837-1840. 
Murray PR, Rosenthal KS, Kobayashi GS, Pfaller MA 2004. Microbiologia médica. 4 ed. Rio de Janeiro: Guanabara Koogan.

NeyndorffHC, Bartel DL, Tufaro F, Levy JG 1990. Development of a model to demonstrate photosensitizer-mediated viral inactivation in blood. Transfusion 30: 485-490.

Osorio YE, Zhao W, Espitia C, Travi BL, Hawel L 2008. Dominant arginase expression in a model of progressive visceral leishmaniasis. FASEB J 22: 860.

Pículo F, Macedo CG, Aandrade SF, Maistro EL 2010. In vivo genotoxicity assessment of nerolidol. J App Toxicol doi: 10.1002/jat.1607. [Epub ahead of print]

Roberts SC, Tancer MJ, Polinsky MR, Gibson KM, Heby O, Ullman B 2004. Arginase plays a pivotal role in polyamine precursor metabolism in Leishmania. J Biol Chem 279: 23668-23678.

Santin RM, Dos Santos AO, Nakamura CV, Dias Filho BP 2009. In vitro activity of the essential oil of Cymbopogon citrates and its major component (citral) on Leishmania amazonensis. Parasitol Res 105: 1489-1496.

Santos DO, Coutinho CER, Madeira MF, Bottino CG, Vieira RT, Nascimento SB, Bernardino A.; Bourguignon SC, Corte-Real S 2008. Leishmaniasis treatment - a challenge that remains: a review. Parasitol Res 103: $1-10$.

Santos LO, Marinho FA, Altoé EF, Vitorio BS, Alves CR, Britto C, Motta MCM 2009. HIV aspartyl peptidase inhibitors intefere with cellular proliferation, ultrastructure and macrophage infection of Leishmania amazonensis. PLOS One 4: 4918.

Schmidt NJ 1979. Cell culture technique for diagnostic virology. In Lennette EH, Schmidt NJ (eds.) Diagnostic procedures for viral and rickettsial infections. New York: American Public Health Association Inc., p. 65139.

Silva JR, Do Carmo DFM, Reis EM, Machado GMC, Leon LL, Silva BO 2009. Chemical and biological evaluations of essential oil with economic value from Lauraceae species. J Brazil Chem Soc 20: 1071-1076.

Ueda-Nakamura T, Mendonça R, Morgado-Dias JA, Maza PK, Dias Filho BP 2006. Antileishmanial activity of Eugenol-rich essential oil from Ocimum gratissimum Parasitol Int 55: 99-105.

Vincendeau P, Gobert AP, Dalouedes S, Moynet D, Mossalayi MD 2003. Arginases in parasitic diseases. Trends Parasitol 19: 9-12.

Wanasen N, Soong L 2008. L-arginine metabolism and its impact on host immunity against Leishmania infection. Immunol Res 41: 15-25.

WHO 2007. Control of leishmaniasis. World Health Organization, http://www.who.int/leishmaniasis/en/ index.html, accessed in January 2011.

\section{*Correspondence}

Rosangela Maria de Araújo Soares

Instituto de Microbiologia Prof. Paulo de Góes, Centro de Ciências da Saúde, Bloco I, Universidade Federal do Rio de Janeiro

21941-590 Rio de Janeiro-RJ, Brazil

rasoares@micro.ufrj.br

Tel.: +55212562 6711/25626513

Fax: +552125626512 